\title{
Analysis and Comparision of Different Spectrum Sensing Technique for IEEE 802.11
}

\author{
Adil Niyaz Makhdoomi ${ }^{1}$, Rashmi Raj ${ }^{2}$ \\ ${ }^{1}$ M.Tech Scholar, ${ }^{2}$ Assistant Professor \\ Department of Electronics and Communication Engineering \\ Universal Institute of Engineering \& Technology, Mohali, Punjab, India
}

\begin{abstract}
The electromagnetic spectrum is natural resources. The use of wireless communication grows day by day but spectrum allocation policies is static, it tends to increase the spectrum scarcity problem. COGNITIVE RADIO refers to advance wireless radio which aims to improve the spectrum utilization by identify unused spectrum from environment. Spectrum sensing proposed the key method of cognitive radio which detects the presence of primary user in licensed frequency band to utilize unused spectrum. There are three categories of spectrum sensing techniques; NonCooperative System, Cooperative System, Interference Based Sensing. The current work aim on the performance analysis of Non-Cooperative System under low and high SNR, validating the result and applied the technique for IEEE 802.11 (WLAN), IEEE 802.16 (WIMAX). To estimate the threshold chi-square equation has been solve and identify no. of detected signal, signal under AWGN with the help of MATLAB software. It has been observed during analysis that energy rises at high SNR under AWGN and under high SNR no. of detected signal decreases gradually when the no. of sample increases. Under low SNR no of detected signal increases when no. of sample increases gradually.
\end{abstract}

Keyword: Cognitive radio, Non-cooperative system, WLAN, WIMAX, AWGN

\section{INTRODUCTION}

The demand of wireless communication grows rapidly. Due to limited spectrum allocation policy, raise a problem named spectrum scarcity. Most of the useful spectrum is allocated to licensed users (e.g. mobile carriers, TV broadcasting companies) that do not utilizes allocation spectrum band in all the

geographical locations all the time. The licensed users are those users who paid licensing fee to the government agencies like Telecom Regulatory Authority of India (TRAI) and Federal Communications Commission (FCC) in the United States. If this unused spectrum is opened for unlicensed user (e.g. private users, short range networks) then it becomes promising solution to spectrum scarcity problem. Some of the examples are Wi-Fi and Bluetooth operating in unlicensed bands.

The cognitive radio is an emerging technology in wireless communication. Cognitive radio is an advanced technique which reduces the problem of spectrum scarcity in electromagnetic spectrum. Spectrum sensing is one of the method which checks the emptiness of primary user allocated to particular frequency spectrum. There are several methods for spectrum sensing for Non-cooperative and cooperative system. Some of the techniques for spectrum sensing for Non-cooperative system are energy detection, matched filter, cyclostationary feature detection.

In this paper we analyze the performance NonCooperative System under low and high SNR, validating the result and applied the technique for IEEE 802.11 (WLAN), IEEE 802.16 (WIMAX).

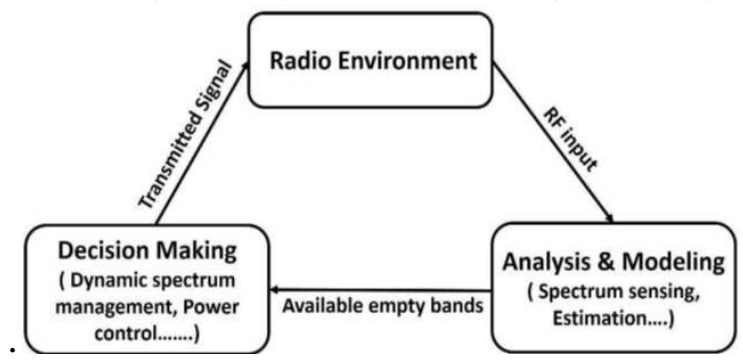

Fig1. Cognitive radio cycle 
Hence to estimate the threshold chi-square equation has been solve and identify no. of detected signal, signal under AWGN with the help of MATLAB software.

\section{THEORETICAL BACKGROUNDS}

The demand of wireless communication grows rapidly. Due to limited spectrum allocation policy, raise a problem named spectrum scarcity .To mitigate the problem of spectrum scarcity we use cognitive radio.

\subsection{Cognitive Radio:}

Cognitive radio is a technology through which unutilized spectrum is detected and if primary user occurs it jump to another unutilized spectrum to avoid the interfering to another primary user or licensed user.

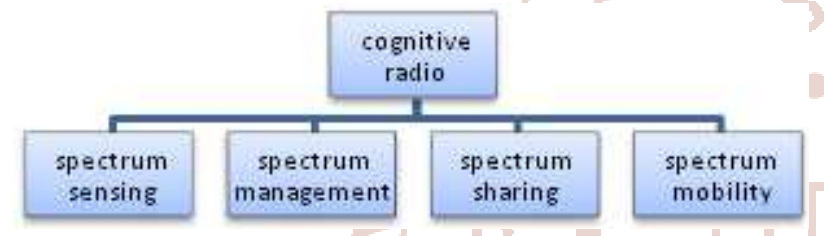

Fig;-2 Functional block diagram of cognitive radio

\subsection{Spectrum Sensing:}

The main objective of spectrum sensing is to identify the spectrum is available for secondary users or not and it also detect the presence of primary users from licensed band. Simply spectrum sensing is a method which determine whether a given frequency band is being used.

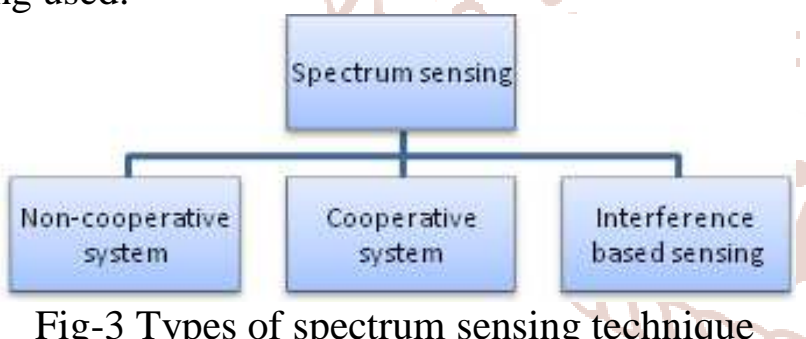

Fig-3 Types of spectrum sensing technique

\subsection{Non- cooperative system:}

In this system no cooperation is allowed during transmission due to lack of communication between sensing terminal that is known as non-cooperative system. Basically it is divided into three parts: Energy detection, Matched filter detection, Cyclostationary detection

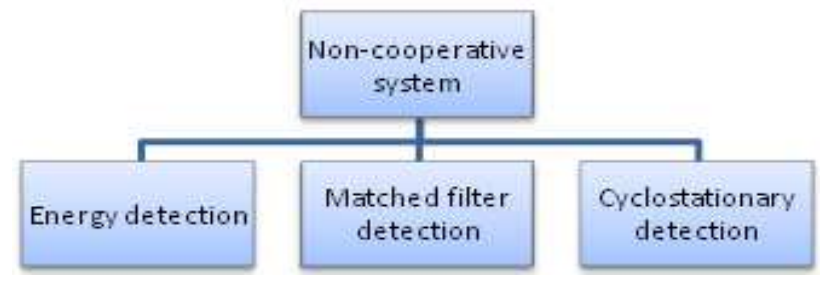

Fig 4- Types of Non-cooperative system

\subsubsection{Energy detection:}

It is a no cooperative detection technique .It simple detection technique because it does not require prior information about structure of signal. Energy detection is based on the principle that, at the reception, the energy of the signal to be detected is calculated. It estimates the presence of a signal by comparing the energy received with a known threshold $\lambda$ derived from the statistics of the noise.

\subsubsection{Matched filter detection:}

Matched filter is a linear filter which used to maximize signal to noise ratio in presence of additive noise. It provides coherent detection. A coherent detector uses the knowledge of the phase of the carrier wave to demodulate the signal.

\subsubsection{Cyclostationary detection:}

MF detection performances better in low SNR condition. But MF requires prior information about signal structure for licensed user detection. So with limited information about signal structure primary user detection can be possible by using cyclostationary feature detection.

\section{PROBLEM FORMULATION:}

\subsection{Energy detection:}

$$
\begin{array}{r}
R(t)= \begin{cases}n(t) & X_{0} \\
s(t)+n(t) & X_{1}\end{cases} \\
E(t)= \begin{cases}\boldsymbol{X}_{\mathbf{0}}, & E<\lambda \\
\boldsymbol{X}_{1}, & E \geq \lambda\end{cases}
\end{array}
$$

Where $R(t)$ is receive signal at each instant ' $t$ ', $n(t)$ is the noise, $\mathrm{s}(\mathrm{t})$ is the detected signal which presence in the network, $X_{0}$ is the no signal transmitted $X_{1}$ is the signal transmitted and $E(t)$ is estimated energy of the received signal.

\subsection{Chai-square distribution equation:}

$$
E \sim\left\{\begin{array}{lc}
N\left(n \sigma_{n}^{2}, 2 n \sigma_{n}^{4}\right) & X_{0} \\
N\left(n\left(\sigma_{n}^{2}+\sigma_{s}^{2}, 2 n\left(\sigma_{n}^{2}+\sigma_{s}^{2}\right)^{2}\right)\right) & X_{1}
\end{array}\right.
$$

Where $\mathrm{n}$ is the number of the samples, $\sigma_{n}^{2}$ is the variance of the noise, $\sigma_{s}^{2}$ is the variance of the received signal $\mathrm{s}(\mathrm{t})$.

$$
P_{f}=Q\left(\frac{\lambda-2 t_{s} w \sigma_{n}^{2}}{\sqrt{4 t_{s} w \sigma_{n}^{4}}}\right)
$$

Where $P_{f}$ is the probability of false alarm, $t_{s}$ is the time symbol or observation time, $\mathrm{w}$ is the bandwidth of the spectrum and $\lambda$ is the threshold value which is determined through this equation.

$$
\lambda=\sqrt{4} t_{s} w \sigma_{n}^{4} Q^{-1}\left(P_{f}\right)+2 t_{s} w \sigma_{n}^{2}
$$


International Journal of Trend in Scientific Research and Development (IJTSRD) ISSN: 2456-6470

3.2.1 Geometrical model

\begin{tabular}{|l|c|c|}
\hline \multicolumn{1}{|c|}{ PARAYETERS } & SYMBOL & VALUE \\
\hline Bandwidth & W & $10^{5}$ \\
\hline Signal to noise ratio & SNR & $30 \& \cdot 30$ \\
\hline Time symbol & t & $10^{2}$ \\
\hline No. of sample & $N$ & $100 \& 200$ \\
\hline Variance of the noise & & $\$ 10^{-2}$ \\
\hline Variance of the signal & & $10^{6}$ \\
\hline
\end{tabular}

\section{RESULTS}

The result obtained as a result of detecting the signal using MATLAB software has been plotted below which has been taken with variable sample size such as 100 and 200 as well as variable energy such as -30 $\mathrm{Db}$ and $30 \mathrm{Db}$

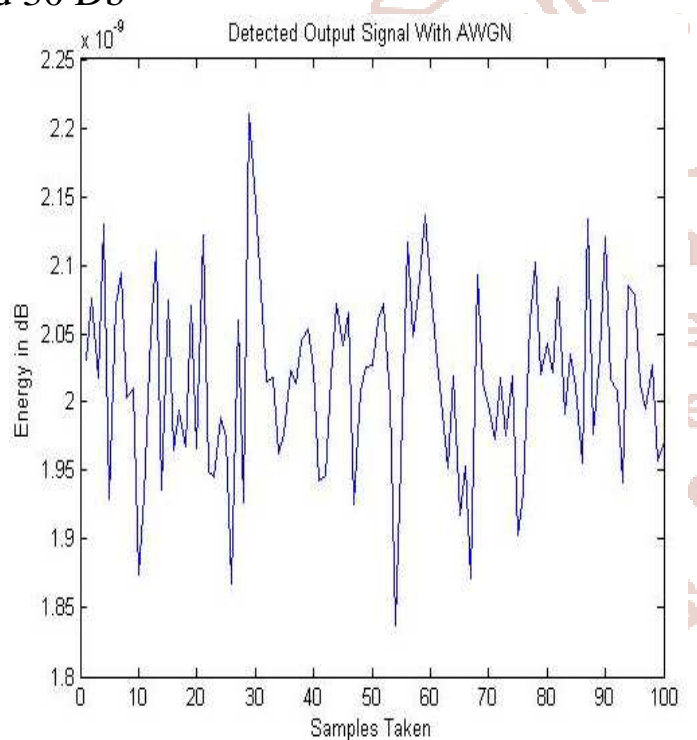

Fig 5 received signal under AWGN

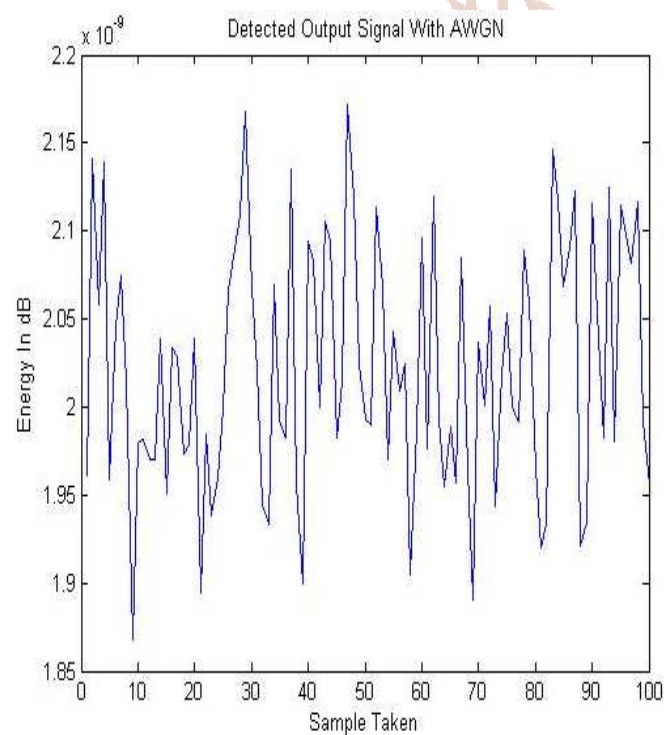

Fig 6 energy at $-30 \mathrm{Db}$ at 100 sample

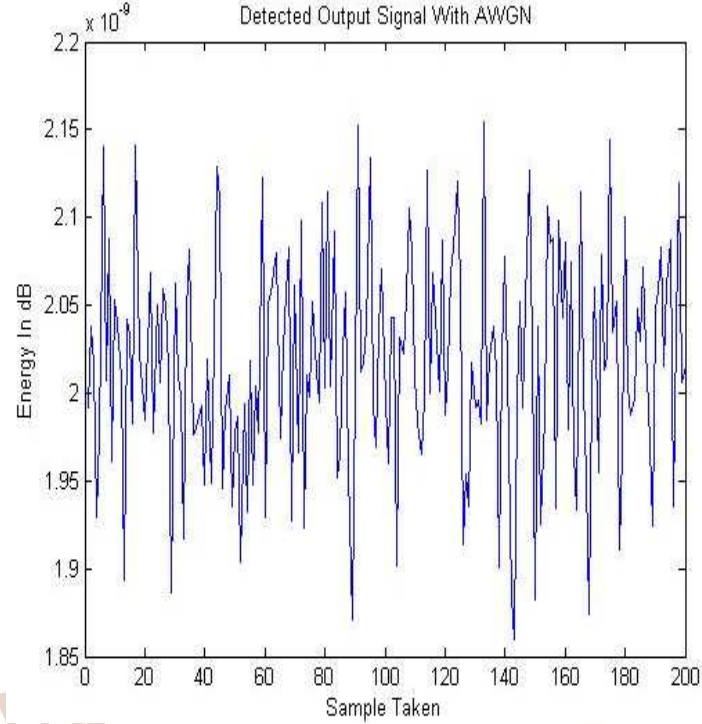

Fig7 energy at $30 \mathrm{db}$ at 200 sample

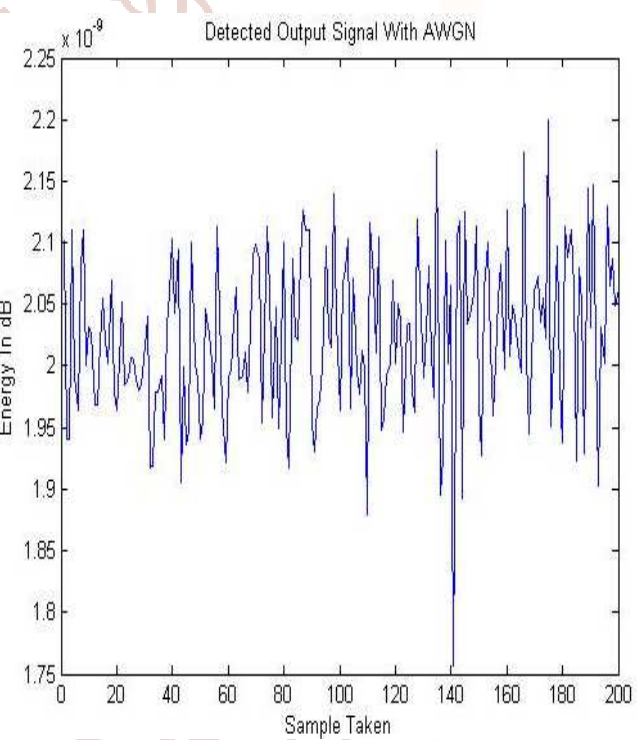

Fig 8 energy at $-30 \mathrm{db}$ at 200 sample

The result of energy detector using MATLAB shows no. of detected signal

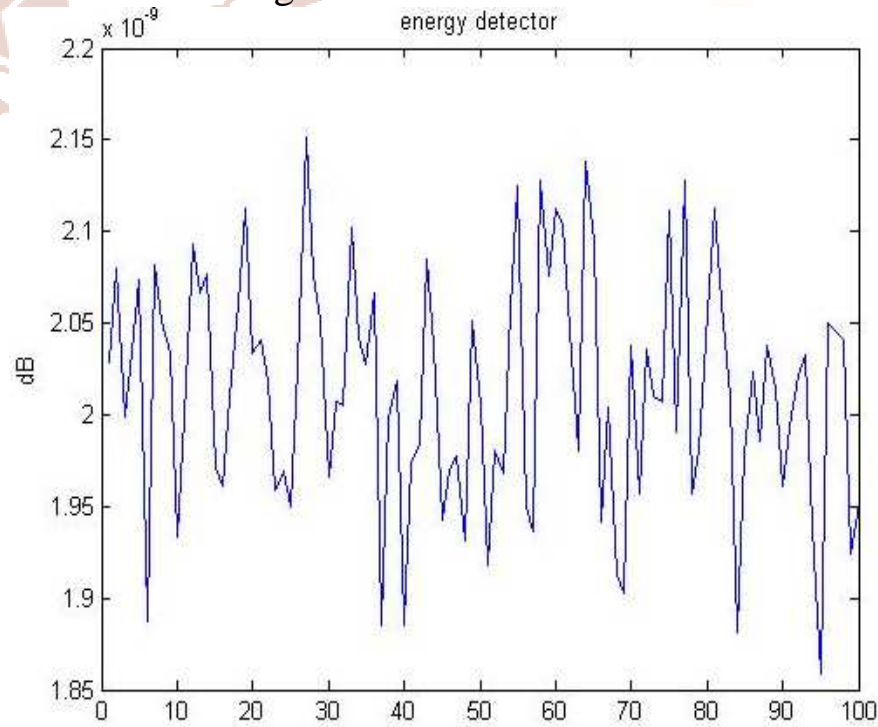

Fig $930 \mathrm{~dB}$ SNR at 100 sample 


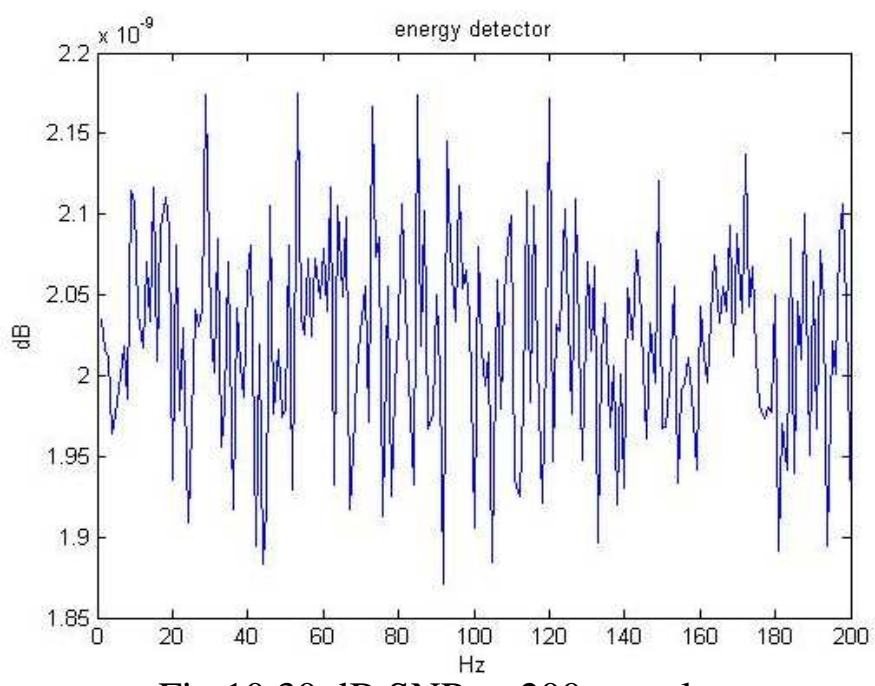

Fig 1030 dB SNR at 200 sample

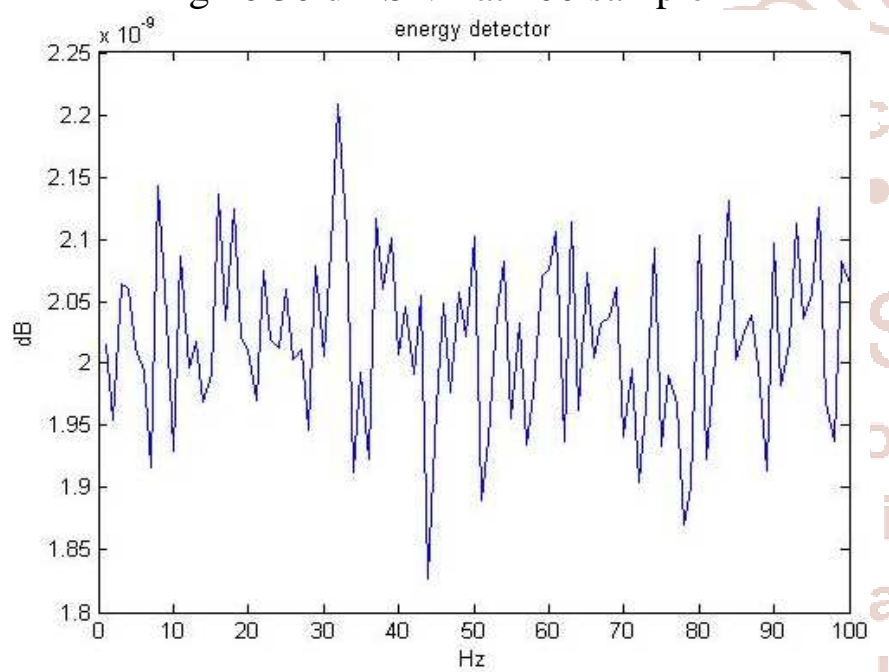

Fig $11-30$ dB SNR at 100 sample

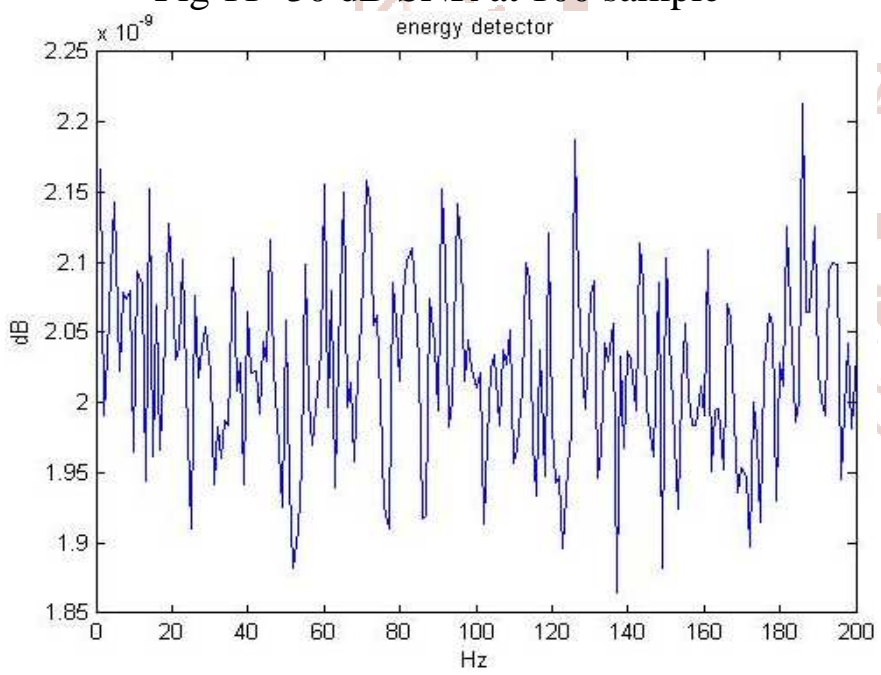

Fig 12: $-30 \mathrm{~dB}$ SNR at 200 sample

\section{CONCLUSIONS}

$>$ Different energy signal at different sample at high and low SNR.

$>$ It also show that under high SNR no. of detected signal decreases gradually when the no. of sample is increases, under low SNR no of detected signal increases when no. of sample increases.

\section{ACKNOWLEDGEMENT}

No work is considered complete unless due indebtedness is expressed to all those, who made the work successful. Concentration, dedication, hard work $\&$ application are essential but not the only factors to achieve the desired goal. There must be supplemented by guidance, assistance and co-operation of people to make it a success. Every complete successful research paper is the result of many hands joined together.

I am thankful to my mentor Er. Rashmi Raj and faculties of our College who have directly or indirectly helped in this Paper. I extremely like to thank my friends and seniors who helped me, who encouraged me to do this. It is warmth and efforts of my friends and well-wishers who have been a source of strength and confidence for me in the endeavour. Finally, thanks to the Almighty who remained with me every time and always helped to direct me in the right direction which could lead me to my goal.

\section{REFERENCES}

1. S. Haykin, "Cognitive radio: brain-empowered wireless communications," Selected Areas in Communications, IEEE Journal on, vol. 23, no. 2, pp. 201-220, 2005

2. Al-Habashna, A. and Dobre, O. A. and Venkatesan, R. and Popescu, D. C., "SecondOrder Cyclostationarity of Mobile WiMAX and LTE OFDM Signals and Application to Spectrum Awareness in Cognitive Radio Systems," Selected Topics in Signal Processing, IEEE Journal of, vol. 6, pp. 26-42, 20

3. M. Subhedar and G. Birajda, "Spectrum Sensing Technique in Cognitive Radio Networks: A Survey," International Journal of Next-Generation Networks(IJNGN), vol. 3, no. 2, pp. 37-51,, June 2011

4. T. Yucek. and H. Arslan, "A Survey of Spectrum Sensing Algorithms for Cognitive Radio applications," IEEE Communications Surveys and Tutorials, vol. 1, no. 1, pp. 116-130, 2009.

5. Sutton P, Nolan K, Doyle 1. Cyclostationary signatures in practical cognitive radio applications. IEEE Journal on Selected Areas in Communications 2008; (26 January):13-24.

6. Dandawate A, Giannakis G, Statistical test for presence of cyclostationarity. IEEE Transactions on Signal Processing 1994; 42(September): 